\title{
Evaluation of solid-liquid interface profile during continuous casting by a spline based formalism
}

\author{
S K DAS \\ Computer Application Division, National Metallurgical Laboratory, Jamshedpur 831 007, India
}

MS received 2 January 2001

\begin{abstract}
A numerical framework has been applied which comprises of a cubic spline based collocation method to determine the solid-liquid interface profile (solidification front) during continuous casting process. The basis function chosen for the collocation algorithm to be employed in this formalism, is a cubic spline interpolation function. An iterative solution methodology has been developed to track the interface profile for copper strand of rectangular transverse section for different casting speeds. It is based on enthalpy conservation criteria at the solidification interface and the trend is found to be in good agreement with the available information in the literature although a point to point mapping of the profile is not practically realizable. The spline based collocation algorithm is found to be a reasonably efficient tool for solidification front tracking process, as a good spatial derivative approximation can be achieved incorporating simple modelling philosophy which is numerically robust and computationally cost effective.
\end{abstract}

Keywords. Continuous casting; solidification; solid-liquid interface; front tracking algorithm; phase change; heat transfer.

\section{Introduction}

The importance of solidification processing in metallurgical operations need not be over emphasized, as the metallurgical structure, distribution of nonmetallic inclusions, micro and macro-segregation as well as mechanical properties of the cast products are intimately associated with the solidification phenomena. Heat transfer and solidification are critical transport phenomena in material processing operations. Solidification carried out properly results in solid with particular physical and mechanical properties. However, uncontrolled or misunderstood solidification process can lead to materials with nonuniform or undesirable characteristics. Casting tend, by their very nature, to be less controlled than the products of crystal growth. They are generally produced on a larger scale, and solidification does not always occur under steady state condition (except in continuous casting) (Taylor 1975; Emlay 1976).

The heat transfer during solidification in continuous casting process strongly influences product quality and process productivity. Therefore, theoretical prediction of solidification front (interface between solid and liquid) which is the indicator of rate of progress of solidification and temperature field in the cast product is of practical industrial significance. The liquid metal with superheat is poured into the mould that has an internal passage through which the primary cooling water is circulated. The primary cooling water is jetted out of the bottom of the mould to impinge directly on the casting surface. Because of this removal of heat from the molten metal, a solidified shell is formed. The shell must be sufficiently thick to contain the remaining liquid and permit continuous withdrawal of the casting.

The characteristic feature of any phase change problem is the coupling of thermal fields in the different phases with free and moving boundaries that not only separate each phase, but also dynamically evolve in transient cases. The free surface and propagating phase front make such problems non-linear. Because of nonlinearity, only a few exact solutions have been developed, and these are limited to simple geometries and boundary and initial conditions. For most practical cases, the solutions are obtained using numerical methods. The characteristic feature of any phase change problem is the coupling of thermal fields in the different phases with free and moving boundaries that not only separate each phase, but also dynamically evolve in transient cases. The free surface and propagating phase front make such problems nonlinear. Because of nonlinearity, only a few exact solutions have been developed, and these are limited to simple geometries and boundary and initial conditions. For most practical cases, the solutions are obtained using numerical methods.

In general the numerical methods for solving the phase change problems can be further classified into two categories: fixed grid approach and transformed grid approach. The fixed grid approach implies that a grid is fixed in space and the interface conditions are accounted for by the definition of suitable source terms in the governing 
equations. In the transformed grid approach, the governing equations and their boundary conditions are cast into a generalized curvilinear coordinate system, so the grid might adapt with the moving freezing front. A comparison between transformed grids and fixed grids on a test problem of the melting of gallium was made by Lacroix and Voller (1990). They have found that if an efficient grid generation can be achieved, the Central Processing Unit (CPU) usage of the transformed grid is very close to that of the fixed grid. Furthermore, a fixed grid can produce accurate predictions with the same order of mesh size as that used by transformed grid. Thus, according to these authors, no conclusive preference can be made for either method. However, the tests covered only a limited range of grid and time resolution; more effort in this direction will be needed to further clarify this issue.

One well known method to deal with phase change problems on fixed grids is the enthalpy-porosity technique. This approach easily permits solutions for substances whose change of phase occurs over a range of temperature (Voller and Prakash 1987; Brent et al 1988). They have adopted this technique in conjunction with the orthogonal control volume based SIMPLE procedure (Patankar 1980) to simulate the two-dimensional convective melting of pure gallium in a rectangular cavity. The enthalpy-porosity technique was validated by comparing its results with the experimental results obtained by Gew and Viskanta (1986). A review of available implicit difference in enthalpy-porosity schemes can be found in Voller (1985). Treatment of the phase change and the associated transport phenomena based on the fixed grid method have been developed to treat quite complicated problems. Bennon and Incropera (1987) have used a volume averaged continuum model with the orthogonal SIMPLE algorithm of Patankar (1980) to investigate solidification of binary, aqueous ammonium chloride solution. Backermann and Viskanta (1988) have also used the same numerical method to solve the complex phase change problems. Shyy and Chen (1990) have combined the enthalpy formulation and a computational scheme based on an adaptive grid in curvilinear coordinates to solve the phase change problems with high Rayleigh and Marangoni numbers, and under both normal and reduced gravity conditions. With relatively finer and improved resolutions, various detailed transport phenomena and interface shapes have been reported. Recently, a turbulence model has been incorporated in this framework to predict the solidification aspects of a continuous casting (Shyy et al 1992) yielding a highly advanced tool for analysing transport phenomena during solidification. Crowley (1983) applied an explicit finite difference scheme to enthalpy formulation and coordinate transformation techniques to study crystal pulling by the Czochralski technique. A numerical solution for melting around a vertically heated cylinder incorporating the effect of natural convection was obtained by Sparrow et al (1977).
They used the coordinate transformation to immobilize the solid-liquid interface and consequently appear to have been the first to apply it to a multidimensional convection-dominated Stefan problem. However, the higher order curvature terms for the interface were neglected, limiting the validity of the method to cases where the radius of the interface varied only slightly. Furthermore, a quasi-steady assumption was invoked where the effect of interface motion was ignored; hence, the method can treat the problem only with very modest phase change rate. Thompson and Szekely $(1988,1989)$ applied this quasisteady approach to predict the phase change problems in a cavity.

Coordinate transformation techniques have been widely used because of the advantage of working with fixed domains (the moving boundaries are immobilized in the transformed coordinates) and a good review in this approach is provided in the work by Crank (1984). However, the simplification obtained by employing coordinate transformations introduces greater complexities into the transformed governing equations. Kim and Kaviany (1990) developed an accurate finite-difference method for phasechange problems with multiple moving boundaries of irregular shape by employing a coordinate transformation that immobilizes moving boundaries and preserve the conservative forms of the original governing equations. The numerical method was first presented for onedimensional phase-change problems (involving large density variation between phases, heat generation, and multiple moving boundaries) and then extended to solve two-dimensional problems (without change of densities between phases). A modified numerical scheme based on non-orthogonal control volume discretization and coordinate transformation technique has been developed and applied to the continuous casting of $\mathrm{Al}-\mathrm{Mg}$ alloy cylindrical ingot under different operating conditions (Das 1993, 1994, 1999; Das and Sarkar 1996).

However, to the best of knowledge of the author no previous work has been reported on usage of spline based collocation formalism exclusively used for tracking of solidification profile during continuous casting of metals and alloys. In the present study, an effort has been made to track the solidification profile numerically by developing a cubic spline based collocation algorithm for continuous casting process. The solidification profile has been modelled for a copper strand of transverse section during continuous casting operation.

\section{Mathematical heat flow model}

During continuous casting process, the temperature distribution of the ingot is independent of time (i.e. steady state operation), and only dependent of spatial coordinates. This amounts to a steady state thermal transport phenomena in continuous casting. The numerical techniques 
applied for solving the thermal transport phenomena during the solidification processing of materials can be broadly classified into two distinct categories:

(i) The single region method (fixed domain): This applies to the thermal transport equation over the complete domain covering both of the phases. The latent heat release is simulated either by appropriate modification of specific heat or a scheme for temperature rise.

(ii) The multiple region method (variable domain): The multiple region method applies to the governing thermal transport equation separately for each phase and involves the coupling of the interface between the phases through interface condition involving heat balance at the interface. Since, the position of the interface is a priori unknown and must be determined as part of the solution, this thermal transport problem may also be designated as free boundary problem.

In general, multiple region method offers better accuracy than that of single region approach. Furthermore, multiple region method is more attractive and powerful because, it can be applied to predict the effects of the fluid flow in the melt or external factors such as rotations and electromagnetic stirring on the process of solidification in an efficient manner. When the concept of multiple region method is applied to the solidification problem, the physical domain of interest $\Omega$ can be subdivided into two sub-domains viz. $\Omega_{\mathrm{l}}(t)$ and $\Omega_{\mathrm{s}}(t)$ which corresponds to liquid and solid regions, respectively. In phase change problems, heat transfer mechanism, which is responsible for supplying the latent heat of fusion at the phase change interface, is of significant importance. For continuous casting of non-ferrous metals e.g. copper or aluminum, $T_{1}>T_{\mathrm{m}}$ and $T_{\mathrm{s}}<T_{\mathrm{m}}$. Here, $T_{1}, T_{\mathrm{s}}$ and $T_{\mathrm{m}}$ are liquidus, solidus and melting temperatures, respectively.

The conservation differential equations of heat transport governing the temperature field $(T)$ in the multiple region framework for the continuous casting process are given in generalized form as:

$$
\begin{aligned}
& C_{1} \rho_{1}\left[\frac{\partial \hat{O}}{\partial t}+\vec{V} \cdot \nabla \hat{O}\right]=\nabla \cdot\left(K_{1} \nabla \hat{O}\right) \\
& C_{2} \rho_{2}\left[\frac{\partial \hat{O}}{\partial t}+\vec{V} \cdot \nabla \hat{O}\right]=\nabla \cdot\left(K_{2} \nabla \hat{O}\right)
\end{aligned}
$$

The subscripts 1 and 2 refer to liquid and solid phases respectively. $C, \rho$ and $K$ are specific heat, density and thermal conductivity, respectively and $V$, the velocity field. If the convection effects in the liquid phase are not significant, the velocity field eventually reduces to casting speed. In the solid phase, however, the velocity field means casting speed $w$ only. For a two-dimensional pseudo-steady state formulation for the longitudinal sec- tion of a continuously cast slab, the following assumptions are made: (i) the casting rate and the withdrawal rate are same and the process is under dynamic equilibrium and (ii) the heat diffusion along the vertical direction (i.e. $z$-direction) is quite small and can be neglected.

The governing heat transfer equation can be written as

$$
\begin{gathered}
C_{\mathrm{P}_{1}} \rho_{1} w \frac{\partial T_{1}}{\partial z}=\frac{\partial}{\partial x}\left(K_{1} \frac{\partial T_{1}}{\partial x}\right) \\
C_{\mathrm{P}_{2}} \rho_{2} w \frac{\partial T_{2}}{\partial z}=\frac{\partial}{\partial x}\left(K_{2} \frac{\partial T_{2}}{\partial x}\right)
\end{gathered}
$$

Here, $w$ is the casting speed and subscripts ' 1 ' and ' 2 ' refer to the liquid and solid phases, respectively.

The associated boundary conditions are given with (generalized) indicial notation as

$$
\begin{aligned}
& -K_{1} \frac{\partial \hat{O}}{\partial x}=h_{j}\left(T-T_{j}\right) \\
& -K_{2} \frac{\partial \hat{O}}{\partial x}=h_{j}\left(T-T_{j}\right)
\end{aligned}
$$

where, $h_{j}$ is the convective heat transfer coefficient on the surface $\Gamma_{j}$ for $j=1$ and 2 and this refers to metal-mould and mould-coolant water thermal regimes. Here, $T_{j}$ is the cooling water temperature. On the top surface, Dirichlet type boundary condition has been invoked, i.e.

$$
\hat{O}(x, z)=T_{\mathrm{c}}>T_{\mathrm{m}}
$$

where, $T_{\mathrm{c}}$ is the casting temperature. For melt-solid interface $\left(\Gamma_{\text {in }}\right)$, the following conditions (known as Stefan's condition) can be formulated

$$
\begin{aligned}
& K_{1} \frac{\partial T_{1}}{\partial x}-K_{2} \frac{\partial \hat{O}_{2}}{\partial x}=\rho_{\mathrm{s}} \Delta C ̧ w \frac{\mathrm{d} x(z)}{\mathrm{d} z} \\
& \hat{O}_{1}=\hat{O}_{2}=\hat{O}_{\mathrm{m}} .
\end{aligned}
$$

$x(z)$ is a function describing the solidification front profile in the longitudinal section of the casting, $\rho_{\mathrm{s}}$ the solid density and $\Delta \mathrm{H}$ the latent heat of freezing. This formulation of solidification thermal model is well known which need not require any further elaboration.

\section{Concept of cubic spline-based collocation procedure}

The segment of the spatial independent variable $x$ in the segment $[a, b]$ can be discretized into $n$ subdivisions as

$$
\Delta x_{i}: a=x_{0}<x_{1}<x_{2} \ldots<x_{i-1}<x_{i} \ldots<x_{n}=b,
$$

where, $\Delta x_{i}=x_{i}-x_{i-1}$. The justification of a spline based collocation algorithm for solving the continuous casting 
problem is briefly discussed here. Let us consider the problem of interpolating a given function to the mesh $\Delta x_{i}$ over several points, which is equivalent to the problem of drawing a smooth curve through a set of $(n+1)$ points. One can use a polynomial of degree $n$ passing through all these points which is not desirable if $n$ is fairly large. Such polynomials are likely to have wild oscillations between grid points. Another alternative is to use interpolation polynomials based on a few nearby points. In this case, the polynomial used is different in different regions and the resulting interpolating function is a piecewise polynomial. If the change of polynomial occurs at only the grid points, this approximation will be continuous, but in general, the derivatives will not be continuous. To ensure that the derivatives of the approximating functions are also continuous, we require additional conditions, and the resulting function with maximum degree of smoothness is referred to as a spline. To make the approximation smoother, polynomials of higher degree over each subinterval can be used. The piecewise cubic interpolation or the cubic spline has become the most popular (Todd 1962; Isaacson and Keller 1966; Kreyszig 1993).

Let the concept be applied for the solution of solidification interface tracking problem and the following expressions are defined

$$
\begin{aligned}
& N_{i}=\left.\frac{d^{2} S(x)}{\mathrm{d} x^{2}}\right|_{x=x_{i}} ; \quad N_{i-1}=\left.\frac{d^{2} S(x)}{\mathrm{d} x^{2}}\right|_{x=x_{i-1}} ; \\
& N_{i+1}=\left.\frac{d^{2} S(x)}{\mathrm{d} x^{2}}\right|_{x=x_{i+1}},
\end{aligned}
$$

where, $S(x)$ is one-dimensional cubic spline function, therefore, its second derivative may be expressed as

$$
\frac{d^{2} S(x)}{\mathrm{d} x^{2}}=N_{i-1} \frac{x_{i}-x}{\Delta x_{i}}+N_{i} \frac{x-x_{i-1}}{\Delta x_{i}},
$$

for $x \in\left[x_{i-1}-x_{i}\right]$. Integrating (12)

$$
\begin{aligned}
\frac{\mathrm{d} S(x)}{\mathrm{d} x}= & -N_{i-1} \frac{\left(x_{i}-x\right)^{2}}{2 \Delta x_{i}}+N_{i} \frac{\left(x-x_{i-1}^{2}\right)}{2 \Delta x_{i}} \\
& +\frac{\hat{O}_{i}-\hat{O}_{i-1}}{\Delta x_{i}}+\frac{\left(N_{i}-N_{i-1}\right) \Delta x_{i}}{6} .
\end{aligned}
$$

Now integrating second time,

$$
\begin{aligned}
S(x)= & N_{i-1} \frac{\left(x_{i}-x\right)^{3}}{6 \Delta x_{i}}+N_{i} \frac{\left(x-x_{i-1}\right)^{3}}{6 \Delta x_{i}} \\
& +\left(\hat{O}_{i-1}-\frac{N_{i-1} \Delta x_{i}^{2}}{6}\right) \frac{\left(x_{i}-x\right)}{\Delta x_{i}}
\end{aligned}
$$

$$
+\left(\hat{O}_{i}-\frac{N_{i} \Delta x_{i}^{2}}{6}\right) \frac{\left(x-x_{i-1}\right)}{\Delta x_{i}}
$$

for $x \in\left[x_{i-1}-x_{i}\right]$. The above expressions are based on the interpolation conditions

$$
S\left(x_{i-1}\right)=T_{i-1} ; S\left(x_{i}\right)=T_{i} ; S\left(x_{i+1}\right)=T_{i+1} .
$$

The left hand and right hand derivative evaluation can be made at the point $x_{i}$ which will be required for boundary condition approximation and may be given as

$$
\begin{aligned}
& \left.\frac{\mathrm{d} S}{\mathrm{~d} x}\right|_{x=x_{i}+0^{+}}=-\frac{\Delta x_{i+1}}{3} N_{i}-\frac{\Delta x_{i+1}}{6} N_{i+1}+\frac{\hat{O}_{i+1}-\hat{O}_{i}}{\Delta x_{i+1}}, \\
& \left.\frac{\mathrm{d} S}{\mathrm{~d} x}\right|_{x=x_{i}-0^{-}}=-\frac{\Delta x_{i}}{3} N_{i-1}+\frac{\Delta x_{i}}{6} N_{i}+\frac{\hat{O}_{i}-\hat{O}_{i-1}}{\Delta x_{i}},
\end{aligned}
$$

where $x_{i}+0^{+}$and $x_{i}-0^{-}$are the right hand and left hand neighbourhood about the location $x_{i}$. The unknown values $\mathrm{N}_{i}$ are determined from the following system of equation

$$
\begin{aligned}
\frac{\Delta x_{i}}{6} N_{i-1}+ & \frac{\Delta x_{i}+\Delta x_{i+1}}{3} N_{i}+\frac{\Delta x_{i+1}}{6} N_{i+1} \\
& =\frac{\hat{O}_{i+1}-\hat{O}_{i}}{\Delta x_{i+1}}-\frac{\hat{O}_{i}-\hat{O}_{i-1}}{\Delta x_{i}},
\end{aligned}
$$

where, $I=1, \ldots, n-1$.

\section{Interface tracking formalism}

It is assumed that in the longitudinal section of the slab, at some location $z=z_{j}$ with an uniform grid spacing $\Delta x$ in the $x$-direction $\left(0=x_{0}+\Delta x<x_{0}+2 \Delta x \ldots<x_{0}+(n-1)\right.$ $\left.\Delta x<x_{0}+n \Delta x=L\right)$ have been determined and the solidification front coincides with point $x_{k+1}=x_{0}+(k+1) \Delta x$. Here $L$ is the lateral dimension of the casting. The subregions of the liquid phase $D_{1}\left(z_{j}\right)$ and solid phase $D_{2}\left(\mathrm{z}_{j}\right)$ correspond to $x \in\left[x_{0}, x_{k+1}\right)$ and $x \in\left(x_{k+1}, x_{n}\right]$ respectively. The notation of square bracket (e.g. '[') stands for closed interval and ordinary parenthesis (e.g. '(') denotes open interval for the independent variable which are standard mathematical notations.

The local temperature field at location $z=z_{j+1}$ will be approximated by two spline functions $S_{1}\left(x, z_{j+1}\right)$ for the domain $D_{1}\left(z_{j+1}\right)$ and $S_{2}\left(x, z_{j+1}\right)$ for the domain $D_{2}\left(z_{j+1}\right)$ respectively. At the solid-liquid interface for $x=x_{k}$, the Stefan condition can be expressed as

$$
\left.K_{1} \frac{\partial S_{1}(x, z)}{\partial x}\right|_{x=x_{k}, z=z_{k}}-\left.K_{2} \frac{\partial S_{2}(x, z)}{\partial x}\right|_{x=x_{k}, z=z_{j+1}}
$$




$$
\begin{gathered}
=\rho_{s} \Delta H w \frac{\mathrm{d} x(z)}{\mathrm{d} z}, \\
S_{1}\left(x_{k}, z_{j+1}\right)=S_{2}\left(x_{k}, z_{j+1}\right)=T_{\mathrm{m}} .
\end{gathered}
$$

Considering that $\Delta z_{j}$ is the difference between two consecutive steps corresponding to the displacement of the solidification profile from $x_{k+1}$ to $x_{k}$, it is assumed that $(\mathrm{d} x / \mathrm{d} z)_{z=z(j+1)}=\Delta x / \Delta z_{j}$. Evaluation of the differential increment $\Delta z_{j}$ can be obtained by the following procedure: (i) it is assumed that solidification front coincides with grid point $x_{k}$, (ii) the differential increment $\Delta z_{j}$ is chosen arbitrarily and (iii) the temperature field at $z=z_{j+1}$ means that $T_{1}\left(x, z_{j+1}\right)$ for $x \in\left[x_{0}, x_{k}\right)$ and $T_{2}\left(x_{k}, z_{j+1}\right)$ for $x \in\left(x_{k}, x_{n}\right]$ on the basis of numerical analysis (which is to be described later in this paper) is calculated. The boundary condition for $x=x_{k}$ can be presented as the associated Dirichlet condition i.e. the second part of the Stefan condition (8)

$$
T_{1}\left(x_{k}, z_{j+1}\right)=T_{2}\left(x_{k}, z_{j+1}\right)=T_{\mathrm{m}} .
$$

The methodology facilitates evaluation of left hand and right hand derivatives i.e. $\mathrm{d} S_{1}\left(x_{k}-0^{-}, z_{j+1}\right) / \mathrm{d} x$ and $\mathrm{d} S_{2}\left(x_{k}+0^{+}, z_{j+1}\right) / \mathrm{d} x$ and next on the basis of the first part of Stefan condition (8), the correction of step $\Delta z_{j}$ is accomplished as given below

$$
\Delta z_{j}=\frac{\rho_{2} \Delta C ̧ w \Delta x}{\left(K_{1} \frac{\mathrm{d} S_{1}}{\mathrm{~d} x}\left(x_{k}-0^{-}, z_{j+1}\right)-k_{2} \frac{\mathrm{d} S_{2}}{\mathrm{~d} x}\left(x_{k}+0^{+}, z_{j+1}\right)\right)} .
$$

For a simplified analysis of the present algorithm, the thermophysical properties of the solid and liquid phases are assumed to be constant. However, this is not a limitation as Kirchoffs transformation can always be used to overcome the problem of variation of thermophysical properties with temperature. The system of (3) and (4) become

$$
\begin{aligned}
& \frac{\partial T_{1}}{\partial z}=\frac{A_{1} \partial^{2} T_{1}}{\partial x^{2}}, \\
& \frac{\partial T_{2}}{\partial z}=\frac{A_{1} \partial^{2} T_{2}}{\partial x^{2}},
\end{aligned}
$$

where, $A=\left(k_{1} / C_{1} \rho_{1} w\right)$ and $A_{2}=\left(K_{2} / C_{2} \rho_{2} w\right)$. Here, $w$ is the casting speed. The numerical approximation of (23) and (24) at a point $\left(x_{i}, z_{j+1}\right)$ can be represented as follows:

$$
\begin{aligned}
& S_{1}\left(x_{i}\right)-T_{i}^{j}=A_{1}\left(M_{i}\right)_{1} \Delta z_{j+1}, \\
& S_{2}\left(x_{i}\right)-T_{i}^{j}=A_{1}\left(M_{i}\right)_{2} \Delta z_{j+1},
\end{aligned}
$$

For $i=0,1,2, \ldots n$ and $j=0,1,2, \ldots p$. Approximation of boundary conditions for $x=x_{0}=0$ and $x=x_{n}=L$ leads to the following equation

$$
\frac{\mathrm{d} S_{1}}{\mathrm{~d} x}\left(x_{0}+0^{+}\right)=0 ;-K_{2} \frac{\mathrm{d} S_{2}}{\mathrm{~d} x}\left(x_{n}-0^{-}\right)=h_{1}\left(s_{2}\left(x_{n}\right)-T\right) .
$$

Here $h_{1}$ is the convective heat transfer coefficient on the surface $\Gamma_{1}$ and $T_{1}$ is the corresponding temperature.

By using (25), (26) and (27)

$$
\begin{aligned}
& S_{1}\left(x_{i}\right)=T_{i}^{j+1}=T_{i}^{j}+A_{1}\left(M_{i}\right)_{1} \Delta z_{j}, \\
& S_{2}\left(x_{i}\right)=T_{i}^{j+1}=T_{i}^{j}+A_{2}\left(M_{i}\right)_{2} \Delta z_{j} .
\end{aligned}
$$

Substituting (28) and (29) in the corresponding expression

$$
\begin{aligned}
\left(\frac{\Delta x}{6}-\frac{\Delta_{j} A_{1}}{\Delta} x\right) M_{i-1}+\left(\frac{2 \Delta x}{3}+\frac{2 \Delta z_{j} A_{1}}{\Delta x}\right) M_{i} \\
+\frac{\left(\frac{\Delta x}{6}-\Delta z_{j} A_{1}\right)}{\Delta x} M_{i+1}=\frac{T_{i+1}^{j}-2 T_{i}^{j}+T_{i-1}^{j}}{\Delta x} .
\end{aligned}
$$

For $i=1,2, \ldots(k-1)$. However, when $x=x_{0}$ and $x=x_{k}$, the pair of expressions become

$$
\begin{aligned}
& \left(\frac{\Delta x}{3}+\frac{\Delta z_{j} A_{1}}{\Delta x}\right) M_{0}+\left(\frac{\Delta x}{6}-\frac{\Delta z_{j} A_{1}}{\Delta x}\right) M_{1}=\frac{T_{1}^{j}-T_{0}^{j}}{\Delta x}, \\
& T_{k}^{j+1}=T_{m} .
\end{aligned}
$$

Similarly, for $D_{2}\left(x, z_{j+1}\right)$, the following expression can be derived.

$$
\begin{aligned}
T_{k}^{j+1} & =T_{m}, \\
\left(\frac{\Delta x}{6}-\frac{\Delta_{j} A_{2}}{\Delta} x\right) M_{i-1}+\left(\frac{2 \Delta x}{3}+\frac{2 \Delta z_{j} A_{2}}{\Delta x}\right) M_{i} & \\
& +\frac{\left(\frac{\Delta x}{6}-\Delta z_{j} A_{2}\right)}{\Delta x} M_{i+1}=\frac{T_{i+1}^{j}-2 T_{i}^{j}+T_{i-1}^{j}}{\Delta x} .
\end{aligned}
$$

For $i=(k+1),(k+2) \ldots(n-1)$.

$$
\begin{aligned}
\left(\frac{\Delta x}{6}-\frac{\Delta z_{j}}{\Delta x}\right) M_{n-1} & +\left(\frac{\Delta x}{3}+\frac{\Delta z_{j} A_{2}}{\Delta x}\right) M_{n} \\
& =T_{n-1}^{j}-T_{n}^{j}-\frac{h_{1}}{k_{2}}\left(T_{n}^{j}-T_{1}\right) .
\end{aligned}
$$

It may be noted that the system of algebraic equations (30), (31), (32), (33), (34) and (35) are characterized by a system of tri-diagonal matrix equation. The popular tri-diagonal matrix algorithm (TDMA) also known as Thomas algorithm has been used to solve the system of equations (Kreyszig 1993). 


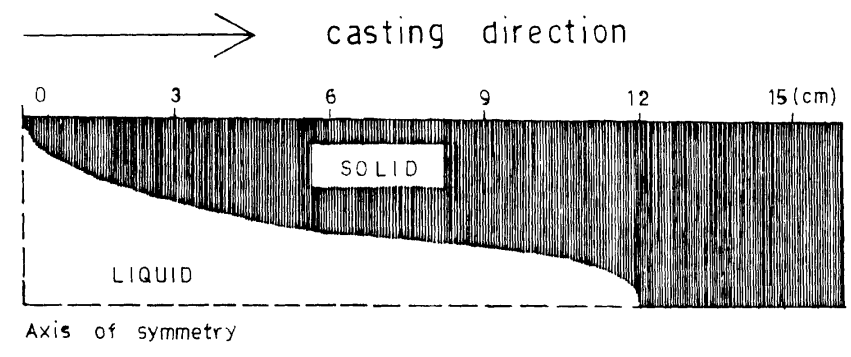

Figure 1. Solidification profile (casting speed $=0.0015 \mathrm{~m} / \mathrm{sec}$ and lateral dimension of the strand $=0.17 \mathrm{~m}$ ).

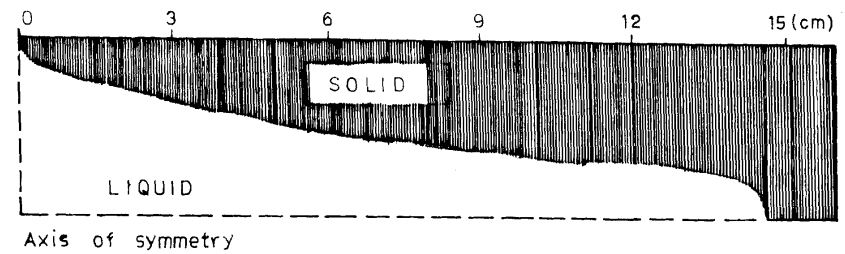

Figure 2. Solidification profile (casting speed $=0.002 \mathrm{~m} / \mathrm{sec}$ and lateral dimension of the strand $=0 \cdot 17 \mathrm{~m}$ ).

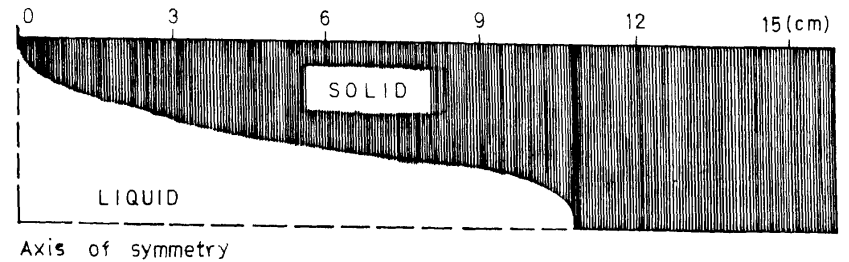

Figure 3. Solidification profile (casting speed $=0.00175 \mathrm{~m} / \mathrm{sec}$ and lateral dimension of the strand $=0.17 \mathrm{~m}$ )

\section{Typical casting applications}

The present formulation is applied to the continuous casting of a rectangular copper strand and the transverse section (vertical section) is considered. The length of the continuous casting mould is taken to be $23 \mathrm{~cm}$. The casting temperature $\left(T_{\mathrm{c}}\right)$ is taken to be $1082^{\circ} \mathrm{C}$. The averaged heat transfer coefficients are $h_{1}=1505 \mathrm{~W} / \mathrm{m}^{2} \mathrm{~K}$ and $h_{2}=$ $905 \mathrm{~W} / \mathrm{m}^{2} \mathrm{~K}$. The dimensions of the lateral section of the cast strand were $0.17 \times 0.17 \mathrm{~m}$. Three different casting speeds have been considered in the analysis. These speeds are $0.0015 \mathrm{~m} / \mathrm{sec}, 0.002 \mathrm{~m} / \mathrm{sec}$ and $0.00175 \mathrm{~m} / \mathrm{sec}$. The number of collocation points in $x$-direction, $n=22$. The casting condition and operating parameters are identical in all these three cases except the casting speed. The interface profile evaluated on the basis of present approach is found to be in good agreement with other numerical investigations by different formalisms (Udaykumar 1993; Shyy 1993). Although, a point to point spatial mapping of the interface profile may not be realizable, but the general trend is quite satisfactory. This is quite evident from the figures $1-3$ and it is self-explanatory.

\section{Conclusion}

The cubic spline function based collocation algorithm has been found to be an efficient method for tracking the solid-liquid interface profile during continuous casting of metals. In this study, an effort has been made to track the solid-liquid interface i.e. the solidification front by developing an effective spline based collocation formalism. The formulation has been applied to the continuous casting of copper strand for different casting speeds under a given operating condition. It is found to be in good agreement with other published investigations with regard to the tracking of solidification interface profiles for metals and alloys which testifies that the algorithmic approach is correct in its entirety.

\section{References}

Bakermann C and Viskanta R 1988 Inst. J. Heat-Mass Transfer 312077

Bennon N D and Incropera F P 1987 Inst. J. Heat-Mass Transfer 302161

Brent A D, Voller V R and Reid K J 1988 Num. Heat-Transfer 13297

Crank J 1984 Free and moving boundary problems (Oxford: Clarendon Press)

Crowley A B 1983 IMA J. Appl. Math. 30173

Das S K 1993 Scand. J. Met. 22198

Das S K 1994 Trans. Ind. Inst. Met. 4757

Das S K 1999 Appl. Therm. Eng. 19897

Das S K and Sarkar A 1996 Comm. Num. Method Engg. 12 657

Emlay E F 1976 Int. Met. Rev. 75

Gew C and Viskanta R 1986 ASME J. Heat-Transfer 108174

Isaacson E and Keller H B 1966 Analysis of numerical methods (New York: Wiley)

Kim C J and Kaviany M 1990 Inst. J. Heat-Mass Transfer 33 2721

Kreyszig E 1993 Advanced engineering mathematics (New York: Wiley)

Lacroix M and Voller V R 1990 Numerical Heat-Transfer B17 25

Patankar S V 1980 Numerical heat-transfer and fluid flow (New York: McGraw-Hill)

Shyy W and Chen H M 1990 Inst. J. Heat-Mass Transfer 33 2545

Shyy W, Pang Y, Hunter G B, Wei D Y and Chen H M 1992 Inst. J. Heat-Mass Transfer 351229

Shyy W, Udaykumar H S and Liang S J 1993 Inst. J. Heat-Mass Transfer 361833

Sparrow E M, Patankar S V and Ramadhyani S 1977 ASME J. Heat-Transfer 99520

Taylor C R 1975 Metall. Trans. B6 389

Thompson M E and Szekely J 1988 J. Fluid Mech. 187409

Thompson M E and Szekely J 1989 Inst. J. Heat-Mass Transfer 321007

Todd J (ed.) 1962 Survey of numerical analysis (New York: McGraw-Hill)

Udaykumar H S and Shyy W 1993 ASME National Heat Transfer Conference, Atlanta (GA, USA: ASME) p. 103

Voller V R 1985 IMA J. Num. Anal. 51205

Voller V R and Prakash C 1987 Inst. J. Heat-Mass Transfer 30 1706 